\title{
Study of the mechanical behavior of plasma-deposited silica films on polycarbonate and steel
}

\author{
A. Hofrichter ${ }^{a)}$ \\ LPICM, Ecole Polytechnique-CNRS, Palaiseau, France \\ A. Constantinescu \\ LMS, Ecole Polytechnique-CNRS, Palaiseau, France \\ S. Benayoun \\ E.N.S.A.M., Angers, France \\ P. Bulkin and B. Drévillon \\ LPICM, Ecole Polytechnique-CNRS, Palaiseau, France
}

In the present study, we deposited amorphous hydrogenated silicon oxide films on polycarbonate, stainless steel, and silicon by plasma enhanced chemical vapor deposition using a low pressure, high density integrated distributed electron cyclotron resonance plasma reactor. Substrate curvature, vibrating slab, and Vickers indentation experiments were used to evaluate the intrinsic stress, the Young modulus of the films, and the composite hardness of the film-substrate system. The indentation experiments were modeled by finite element analysis and the calculated values were compared to experimentally measured hardness values. A reasonable accordance with the experiment was found both for stainless-steel and polycarbonate substrates, indicating that the modeling is valid and may be used to enhance the interpretation of the indentation experiments. The calculations show an important bending of the film in the noncontact region in the case of a Vickers indentation on a coated polycarbonate sample. The analysis of the thus-induced strain distribution in the coating indicates that the measured diagonal might be overestimated and not representative of the real contact area. The calculations indicate that the yield limit of the plasma-deposited silica films is of about $4 \mathrm{GPa}$.

\section{INTRODUCTION}

Polymers are increasingly used to replace more traditional engineering materials such as glass, metals, and ceramics. There is considerable interest in using silicon-based thin films to enhance functional characteristics such as scratch and abrasion resistance or barrier properties. The mechanical properties of film and substrate and the mechanical behavior of the film-substrate composite are of prime importance for such applications. Previous studies focused on the mechanical properties of plasma enhanced chemical vapor deposition (PECVD) deposited films on a nanosized scale, ${ }^{1}$ nevertheless, the macroscopic mechanical properties of the filmsubstrate system were, up to now, mainly studied from a phenomenologic point of view. ${ }^{2,3}$ In this work, we studied silica coated polycarbonate with macroindentation tests and finite-element modeling (FEM) of the mechanical behavior in order to get quantitative information about the hardness of the thin film/substrate composite. As the interpretation of macroindentation tests of hard coatings on soft substrates is particularly delicate, we studied also the mechanical behavior of silica thin films on stainless steel to see if our model can cope with two completely different substrate materials.

${ }^{a)}$ Electronic mail: hofricht@poly.polytechnique.fr

\section{EXPERIMENT}

\section{A. FEM analysis}

In this study the indentation of the sample by a Vickers pyramidal indenter has been modeled using the FEM code CASTEM 2000 (Refs. 4-6) through a two-dimensional (2D) axisymetric indentation of a cylinder by a highly rigid cone. In their analysis of 2D and three-dimensional (3D) models, several authors ${ }^{7,8}$ found that a tip angle of approximately $70^{\circ}$ for the 2D cone leads to a displaced volume of matter similar to that of Vickers or Berkovich pyramidal indenters and provides similar indentation curves.

The contact between the indenter and the mesh has been considered frictionless. In the case of conical indenters this is justified by numerical results obtained by others. ${ }^{8-10}$ One can remark that the friction plays an important role in the case of spherical indenters. ${ }^{11}$ In order to simulate the indentation experiments, characterized by a ratio of film thickness to indentation depth of approximately 1:0.1 and 1:3 for nano- and macroindentation, respectively, two different types of meshes with 54 and 9 (see Figs. 1 and 7) elements, respectively, in the layer have been used. They have been designed in order to permit a high mesh refinement in the contact area and to span a large domain, typically, 15-50 times larger than the indentation depth. All meshes had between 2000 and 4000 linear quadrangular elements and between 2000 and 5000 nodes. 


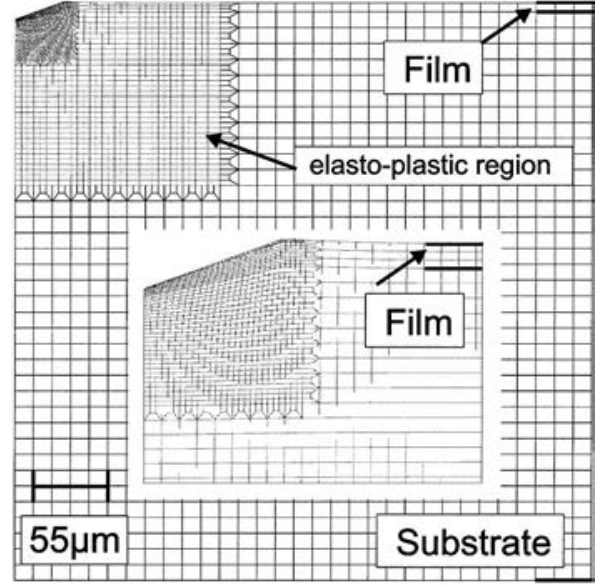

FIG. 1. Deformed finite-element mesh for macroindentation with a zoom of the contact zone.

As a consequence of the large displacements induced by the indenter, the computations have been done under the hypothesis of finite displacements and small strains using a mesh actualization at each load step. This hypothesis is not valid at the tip of the indentor where plastic deformation can attain $200 \%$ on some elements and where the small strain behavior is largely surpassed. This phenomenon is, however, always confined in a very small region at the tip of the indenter.

The material behavior has been considered as elastoplastic with an isotropic hardening ${ }^{12}$ and the material parameters are presented in Table I. It is important to remark that a change to a kinematic hardening or a small variation of the coefficients did not influence the global behavior of the film-substrate structure.

The mechanical behavior of silica has been assumed identical in traction and compression. However, it is well known that silica can be better characterized by plastic behavior in compression and a brittle one in traction and that the limit yield stresses can be very different. This was unfortunately not possible during these computations. Nevertheless, two different values for the plasticity coefficients have been chosen in order to activate the dissipation phenomena at different stress levels. The validity of the constitutive assumption of $\mathrm{SiO}_{2}$ films will stem from the results presented in the next section.

The hardness $H_{v}$ has been computed according to $H_{v}$ $=F / A$, where $F$ represents the resultant force and $A$ the projected contact area obtained from the contact radius in the deformed configuration. The resultant force has been computed using the computed nodal forces or the Lagrange multipliers obtained directly in the contact conditions. The difference between the two methods provided a relative error smaller than $10^{-4}-10^{-6}$.

The computations have been performed on a $400 \mathrm{MHz}$ Intel PC and 100 load increments with about five internal iterations at each step, corresponding to contact and plasticity, took typically $40 \mathrm{~min}$ of CPU time. The use of elastic super elements on the exterior boundary of the domain reduced the number of active nodes by $400-600$ and, consequently, the computed time by $30 \%$.

\section{B. Film deposition and measurements}

The thin-film depositions were carried out in an integrated distributed electron cyclotron resonance (IDECR) microwave excited high density plasma reactor scalable for large area deposition. The system is described in detail in Ref. 13 and allows the deposition of dense stoichiometric silica with growth rates as high as $46 \AA / \mathrm{s}$ at room temperature. ${ }^{14}$ The films were deposited from a mixture of $\mathrm{SiH}_{4}$ and $\mathrm{O}_{2}$ on 1 in. $\times 1$ in. extruded GE Lexan polycarbonate and $316 \mathrm{~L}$ stainless-steel substrates. The microwave power was $100 \mathrm{~W}$ and the pressure was set at 1.14 mTorr. During deposition the substrates were kept at floating potential, the substrate holder was not heated intentionally, and the substrate temperature stayed below $50{ }^{\circ} \mathrm{C}$. The film thickness and refractive index were measured in situ at an angle of $57.8^{\circ}$ on a spectroscopic phase modulated ellipsometer (UVISEL ISA Jobin-Yvon Sophie).

TABLE I. Values of the mechanical constants used for the FEM calculations.

\begin{tabular}{|c|c|c|c|c|c|}
\hline Material & $\begin{array}{c}\text { Young modulus } \\
E(\mathrm{GPa})\end{array}$ & $\begin{array}{c}\text { Yield limit } \\
\sigma_{y}[\mathrm{GPa}]\end{array}$ & \multicolumn{2}{|c|}{$\begin{array}{c}\text { Strain hardening } \\
H(\mathrm{MPa})\end{array}$} & $\begin{array}{c}\text { Poisson coefficient } \\
\nu\end{array}$ \\
\hline $\begin{array}{l}316 \mathrm{~L} \\
\text { (Ref. 15) }\end{array}$ & 170 & 340 & $\begin{array}{l}\boldsymbol{\epsilon} \\
0.002 \\
0.1\end{array}$ & $\begin{array}{r}\sigma \\
\sigma \\
400 \\
1350\end{array}$ & 0.3 \\
\hline $\mathrm{PC}^{\mathrm{a}}$ & 2.54 & 40 & \begin{tabular}{l}
\multicolumn{1}{c}{$\boldsymbol{c}$} \\
0.016 \\
0.038 \\
0.059 \\
0.1
\end{tabular} & $\begin{array}{c}\sigma \\
40 \\
50 \\
56 \\
58\end{array}$ & 0.3 \\
\hline $\begin{array}{l}\mathrm{SiO}_{2} \\
\text { (Ref. 16) }\end{array}$ & $50^{\mathrm{a}}$ & 1 or 4 & & & 0.25 \\
\hline
\end{tabular}

${ }^{\mathrm{a} D e t e r m i n e d ~ b y ~ e x p e r i m e n t . ~}$ 


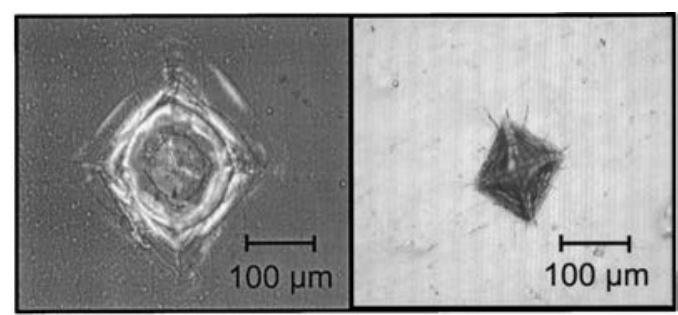

FIG. 2. Images of the Vickers indent on PC (left) and stainless steel (right).

The Vickers indentation tests were performed with standard Vickers indenters at loads between 10 and $1000 \mathrm{~g}$ on bare and coated substrates. For each indent the diagonal was measured by optical microscopy. Particular care was taken to determine the diagonal of the indent, which proved to be difficult in some cases, especially for coated polycarbonate (PC) substrates (Fig. 2). The size of the indent was averaged over the two diagonals of the indenter and each indent was repeated ten times. Penetration depth and Vickers hardness were calculated using standard formulas.

The stress measurements were performed by measuring the bending of small rectangular $c$-Si slabs $(0.7 \times 2.5 \mathrm{~mm})$ of 100 and 111 orientation with a Sloan Dektak III profilometer before and after deposition. The Young moduli of the film and the bare polycarbonate substrate were determined by the vibrating slab technique.

\section{RESULTS AND DISCUSSION}

\section{A. Film and material characterization}

The thickness of the films was $5.50 \pm 0.05 \mu \mathrm{m}$ as determined by ellipsometry. The films had a refractive index of $n=1.456$, identical to that of thermal silica and had a hydrogen content of less than $4 \% .{ }^{14}$ The films had compressive stress of about $(70 \pm 15) \mathrm{MPa}$, which was found to be independent of processing power.

The Young modulus of the bare polycarbonate sample was determined as $2.54 \mathrm{GPa}$, whereas the Young modulus of the $\mathrm{SiO}_{2}$ layers was determined to be $50 \pm 2 \mathrm{GPa}$ (Table I ${ }^{15,16}$ which is well in accordance with the literature values. The traction curve of the bare polycarbonate sample was recorded and the measured strain-stress curve used for determining the isotropic hardening of the substrate.

\section{B. Macroindentation}

The numerical and experimental values of the Vickers hardness obtained for the macroindentation on coated and uncoated stainless steel are displayed in Fig. 3. For highindentation depths the hardness of the film/substrate system tends, as expected, towards the hardness of the bare substrate. The numerical values $(1500 \pm 50 \mathrm{MPa})$ are in reasonable agreement with the experiment for the bare substrate $(1600 \pm 100 \mathrm{MPa})$. In the case of the film/substrate system only the numerical simulation corresponding to a yield stress $\sigma_{y}$ of $4000 \mathrm{MPa}$ for the silica coating are in agreement with the measurements. One can observe that for small indenta-

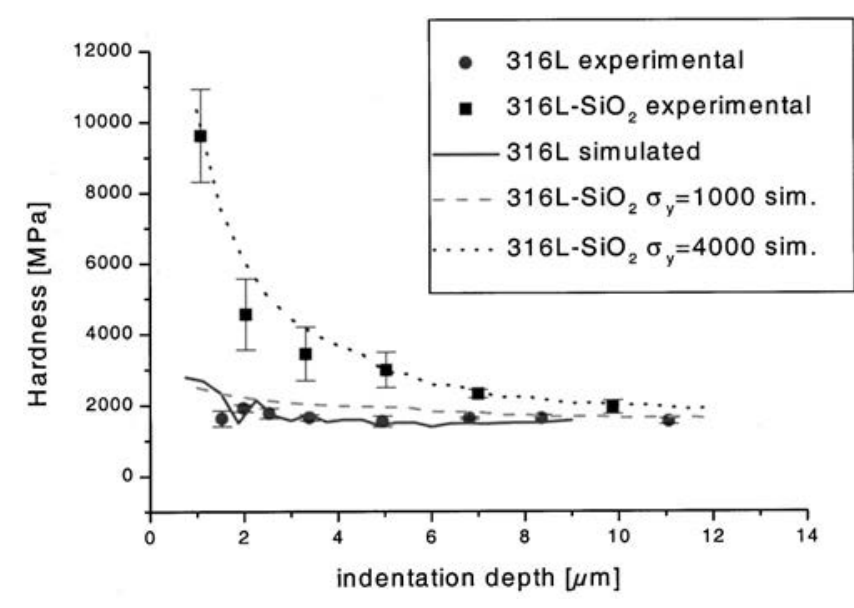

FIG. 3. Computed and measured hardness for coated and uncoated stainless steel vs indentation depth.

tion depths the system shows a high hardness, corresponding essentially to the hardness of the pure film of about $10 \mathrm{GPa}$. This value is higher but of the same order of magnitude as hardnesses reported for similar silica coatings in the literature. $^{1,10}$

In order to effectively examine the measurements on polycarbonate, we must first compare the indentation depth on polycarbonate and steel substrates for an identical contact radius (Fig. 4). The total indentation depth $\delta_{T}$ can be split in two parts: the contact indentation depth $\delta_{C}$ and the structural indentation depth $\delta_{S}\left(\delta_{T}=\delta_{S}+\delta_{C}\right)$. The structural indentation depth characterizes the elastic flexure bending of the film and is related to the ratio of the Young moduli between substrate and film. One can remark that the structural indentation depth is much lower in the case of the stainless-steel substrate. In the case of the polycarbonate the structural indentation depth represents a large part of the computed indentation depth $\delta_{T}$. Therefore, the computed values for the film on the polycarbonate substrate must be plotted as a function of contact indentation depth $\delta_{C}$.

The Vickers hardness values for the coated and uncoated polycarbonate are plotted in Fig. 5. The experimental values of hardness of the coated system tend to a limit of $220 \pm 20$ $\mathrm{MPa}$, whereas the hardness value of the bare polycarbonate is $140 \pm 10 \mathrm{MPa}$.

The computed values of hardness for the coated system are generally larger than the experimental ones. This can be explained by the cracking of the film, which would induce a larger observed indentation diagonal. In order to understand

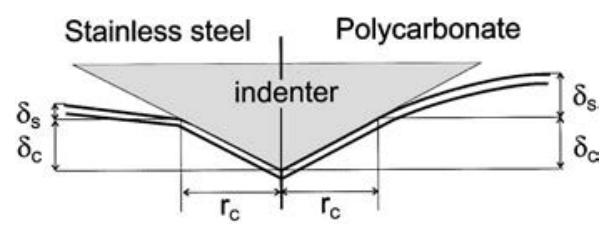

FIG. 4. Schematic diagram of the indentation on coated PC and steel substrates for identical contact area. $r_{C}$ represents the contact radius, $\delta_{s}$ the structural indentation depth, and $\delta_{C}$ the contact indentation depth. 


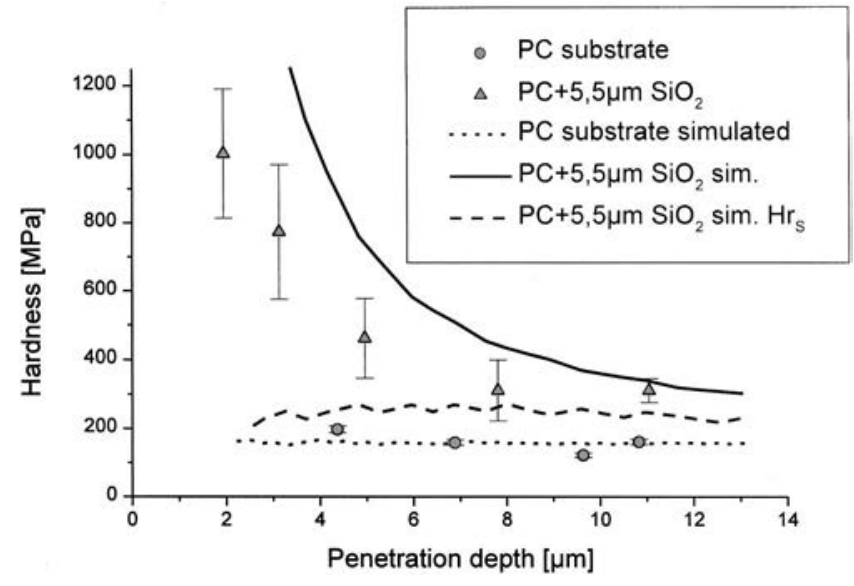

FIG. 5. Computed and measured hardness for coated and uncoated polycarbonate vs indentation depth.

where the film might crack, let us first observe that the bending of the film induces traction in the upper part of the film outside the contact zone. This traction is represented by the radial stress component $\sigma_{r r}$. It is important to understand why this phenomenon is not as strong in the case of a stainless-steel substrate.

If we plot $\sigma_{r r}$ for identical structural indentation depth, or equivalently, the same contact radius along the radius of the sample (Fig. 6), we observe that the maximum lies near the contact radius in the case of stainless steel, whereas it is located far outside the contact region in the case of polycarbonate. This suggests that cracking occurs for the polycarbonate substrate far outside the contact region and that the experimentally observed indentation diagonal will lie somewhere between the computed contact radius and the radius $r_{s}$ of maximal $\sigma_{r r}$. Representing now the computed hardness $H r_{s}$ using the radius $r_{s}$ we remark that the experimental values are well bounded by the two computed hardnesses (Fig. 5).

For high-indentation depth the computed $H_{v}$ tends towards $230 \pm 15 \mathrm{MPa}$, well in accordance with the experimen-

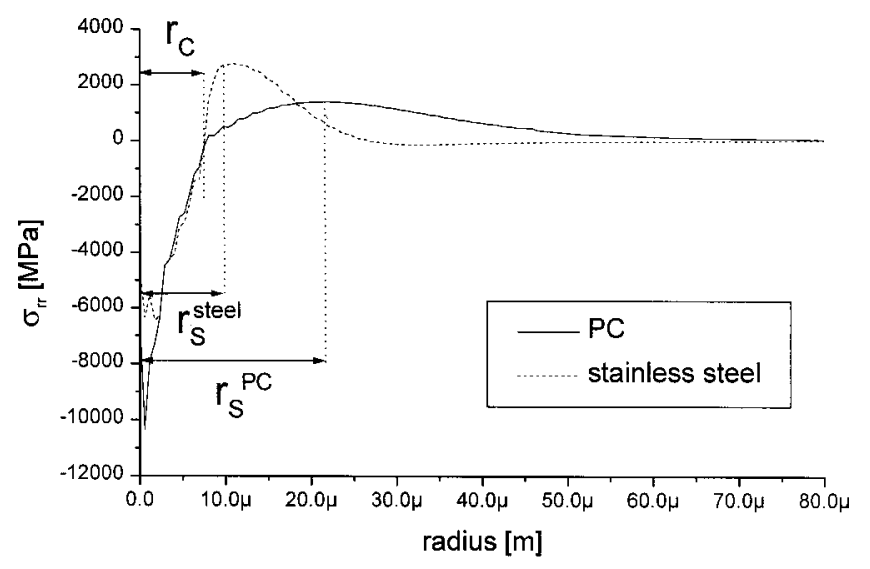

FIG. 6. Radial stress component $\sigma_{r r}$ in the silica film for polycarbonate and steel substrates vs the distance of the tip.

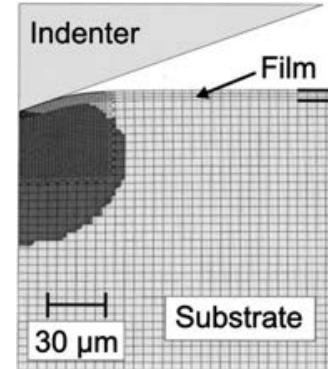

(a)

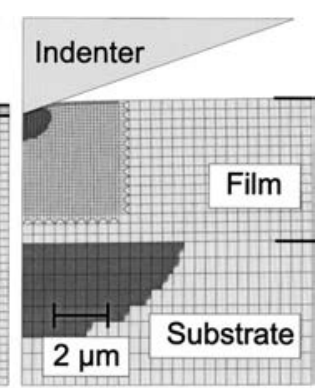

(b)
FIG. 7. Indenter, plastic deformed zone (dark gray), and elastoplastic finite elements (light gray) in the case of macro- (a) and nano- (b) indentations.

tal results. In this case, the correction between $r_{C}$ and $r_{S}$ is of about $5 \mu \mathrm{m}$ for a radius of about $35 \mu \mathrm{m}$ which lies within the experimental error.

For low indentation depths, the computed hardness values tend to the expected value of $10 \mathrm{GPa}$, as in the case of the stainless-steel substrate. For these indentation depths, the structural bending is low and the $\sigma_{r r}$ stress stays under the threshold value responsible for the failure of the coating. Therefore, it does not make sense to talk about $r_{S}$ and the corresponding hardness.

It is obvious that for intermediate indentation depth a more sophisticated model, taking into account the precise fracture behavior of the $\mathrm{SiO}_{2}$ layer and related to precise diagonal measurements, would be necessary in order to simulate the measured values.

\section{Nanoindentation}

Figure 7 compares the plastically deformed area in the film and substrate for Vickers and nanoindentation. In the case of nanoindentation, we observe that the plastic deformation in the film is confined in a small region under the tip of the indenter. In the substrate, the size of the plastically deformed region depends highly on the nature of the substrate. In the coating, the mechanical field distribution is practically independent of the nature of the substrate.

Unfortunately, no experimental values were available at the moment to validate the nanoindentation calculations. The computed hardness values were up to indentation depths smaller than $20 \%$ of the film thickness, independent of the nature of the substrate. This is in accordance with the experiments reported in the literature. ${ }^{3}$ For a film yield limit $\sigma_{Y}$ $=4 \mathrm{GPa}$, the hardness was about $8 \mathrm{GPa}$. These values fit well with the values obtained in the macroindentations on stainless steel for small indentation depths. In the case of the polycarbonate substrate, the smallest indentation depth in our macroindentation experiments is with $2 \mu \mathrm{m}$ about half of the film thickness, and therefore, not low enough to be representative of the film hardness alone.

\section{CONCLUSION}

We studied the macroindentation of silica thin films on stainless-steel and polycarbonate substrates. The indentation experiments were simulated by finite-element analysis and a 
reasonable accordance was found between the experiments and the simulations. The results indicate that the yield limit of the plasma-deposited silica films is of about $4 \mathrm{GPa}$. Further nanoindentation measurements would be necessary to obtain a more precise description of the mechanical properties of the film. The numerical analysis showed that due to film cracking the observed diagonals might be overestimated and not representative of the real contact area.

These simulations show that the finite-element analysis can be effectively used to better understand the mechanical aspects of indentation tests at both the nano- and macrolevel and that experimental hardness values can be computed with reasonable precision. More precisely, it has also been shown that cracking of the film could be predicted by FEM analysis and, therefore, enhance the interpretation of measurements.

These findings may form a basis for using FEM analysis to determine design specifications for protective coatings on polymers and similar single and multilayer structures, although further verification of the model will be necessary.

\section{ACKNOWLEDGMENTS}

The authors would like to thank Nicolas Tardieu for discussing some aspects of the numerical computations, Jean-
Denis Touzalin for performing the indentation tests, and Jean Cristoph Eytard for the polycarbonate traction curve. The authors are grateful for the financial support of Air Liquide, Sekurit Saint Gobain, and from CEE Contract No. ERBIC15CT970706.

${ }^{1}$ U. Beck, D. T. Smith, G. Reiners, and S. J. Dapkunas, Thin Solid Films 332, 164 (1998).

${ }^{2}$ D. Rats, V. Hajek, and L. Martinu, Thin Solid Films 340, 33 (1999).

${ }^{3}$ A. M. Korunsky, M. R. McGurk, S. J. Bull, and T. F. Page, Surf. Coat. Technol. 99, 171 (1998).

${ }^{4}$ CASTEM2000, a finite-element code, http://www.castem.org:8001

${ }^{5}$ A. Combescure, A. Hoffman, and P. Pasquet, in Finite Element System, edited by C. A. Brebbia (Springer, Berlin, 1982).

${ }^{6}$ A. Constantinescu et al., in Object Oriented Methods for Interoperable Scientific and Engineering Computing, edited by M. E. Henderson, C. R. Anderson, and S. L. Lyons, SIAM Proceedings, Philadelphia, PA (1999). ${ }^{7}$ A. K. Bhattacharya and W. D. Nix, Int. J. Solids Struct. 24, 1287 (1988). ${ }^{8}$ M. Lichinchi et al., Thin Solid Films 312, 240 (1998).

${ }^{9}$ A. K. Bhattacharya and W. D. Nix, Int. J. Solids Struct. 28, 1047 (1991).

${ }^{10}$ T. A. Laursen and J. C. Simo, J. Mater. Res. 7, 618 (1992).

${ }^{11}$ M. R. Begley et al., Int. J. Solids Struct. 38, 2773 (1999).

${ }^{12}$ J. C. Simo and T. R. Huges, Computational Inelasticity (Springer, Berlin, 1998).

${ }^{13}$ P. Bulkin et al., Thin Solid Films 308-309, 63 (1997).

${ }^{14}$ A. Hofrichter et al., Appl. Surf. Sci. 142, 447 (1999).

15 C. D'Anjou and L. Valin, Projet en Laboratoire, ENSTA, Paris (1999).

${ }^{16}$ Fischer-Cripps and Lawn, J. Am. Ceram. Soc. 79, 2609 (1996). 